\title{
Inverse Halftoning Based on Bayesian Theorem
}

\author{
Yun-Fu Liu ${ }^{1}$, Jing-Ming Guo ${ }^{2, *}$, and Jiann-Der Lee ,** $^{1, *}$ \\ ${ }^{1}$ Department of Electrical Engineering \\ Chang Gung University \\ Tao-Yuan, Taiwan \\ yunfuliu@gmail.com, jdlee@mail.cgu.edu.tw \\ ${ }^{2}$ Department of Electrical Engineering \\ National Taiwan University of Science and Technology \\ Taipei, Taiwan \\ jmguo@seed.net.tw
}

\begin{abstract}
In this work, a method which can generate high quality inverse halftone images from halftone images is proposed. This method uses least-mean-square (LMS) trained filters to establish the relationship between the current processing position and its corresponding neighbor positions in each kind of halftone image. This includes direction binary search (DBS), error diffusion, dot diffusion, and ordered dithering. After which, the support region which is used for features extracting can be obtained by relabeling the LMS-trained filters by order of importance. Two features are used in this work: 1) the probability of black pixel occurrence at each position in the support region, and 2) the probability of mean occurrence which is obtained from all pixels in the support region. According to these data, the probabilities of all possible grayscale values appearance at current processing position can be obtained by Bayesian theorem. Consequently, the final output at this position is the grayscale value with highest probability. Experimental results show that the image quality and memory consumption of the proposed method are superior to Mese-Vaidyanathan's method.
\end{abstract}

Keywords: Halftoning, inverse halftoning, Bayesian theorem, halftoning classification, and error diffusion.

\section{Introduction}

Digital halftoning [1] is a technique for converting grayscale images into halftone images. These halftone images resemble the original images when viewed from a distance due to the low-pass filtering nature of the human visual system (HVS). The technique is used widely in computer print-outs, printed books, newspapers and magazines, as they are mostly constrained to the black-and-white format (with and without ink). Another major application of digital halftoning is color quantization with a restricted color palette. Halftoning methods include ordered dithering (OD) [1], dot diffusion (DD) [2]-[3], error diffusion (EDF) [4]-[8] and iteration-based halftoning: direct binary search (DBS) [9]-[10].

${ }^{*}$ Member IEEE.

T. Wada, F. Huang, and S. Lin (Eds.): PSIVT 2009, LNCS 5414, pp. 130-142, 2009.

(C) Springer-Verlag Berlin Heidelberg 2009 
Inverse halftoning is a conjugate technique to halftoning, which restores the halftone images to grayscale images. This technique can be used for certain applications such as image enhancement or compression. For Chang-Yu's inverse halftoning [11], the classification procedure for different kinds of halftone images takes priority over the algorithm of inverse halftoning as they want to increase the resulting quality of inverse halftone images. First they transform the halftone images into its Fourier spectrum in order to separate different halftone images. However, the complexity is high. Hence, the method is replaced by the cooperation between one-dimensional correlation and three-layer back propagation neural network. For inverse halftoning, the least-mean-square (LMS) adaptive filtering algorithm is used.

Mese-Vaidyanathan's inverse halftoning [12] is a look-up table (LUT) based method. The resulting images have clear edges and this method is computationally efficient. In addition, the memory consumption is based on the number of halftone value combinations of all pixels in the referenced region. Consumption increases by $2^{L}$, where the $L$ denotes the referenced number of pixels.

In this work, the concept of Chang-Yu's classification for different kinds of halftoning techniques was adopted. There are also two different features taken into consideration in this work, which are 1) the probability of black pixel occurrence at each position in referenced region, and 2) the probability of mean occurrence which is obtained from all pixels in referenced region. The image quality obtained from the proposed method is superior to Mese-Vaidyanathan's inverse halftoning.

\section{Performance Evaluation}

In this section, the performance evaluation, peak single-to-noise ratio (PSNR) employed in this work is defined. For an image of size $P \times Q$, the quality evaluation of grayscale images is defined as,

$$
P S N R=10 \log _{10}\left(\frac{P \times Q \times 255^{2}}{\sum_{i=1}^{P} \sum_{j=1}^{Q}\left[\sum_{m, n \in G F} \sum \operatorname{coe}_{m, n}\left(\text { ori }_{i+m, j+n}-i h_{i+m, j+n}\right)\right]^{2}}\right),
$$

where the variables $o r i_{i, j}$ and $i h_{i, j}$ denote the grayscale value of current processing position $(i, j)$ on original image $(O r i)$ and inverse halftone image $(I H)$, respectively. The variable $\operatorname{coe}_{m, n}$ denotes the coefficient of corresponding position $(m, n)$ in Gaussian filter, and notation $G F$ denotes the Gaussian filter. In this work, $\operatorname{coe}_{m, n}$ is obtained by the following equation of 2-D Gaussian distribution and the size of $G F$ is set to 7x7.

$$
\operatorname{coe}_{m, n}=\frac{1}{2 \pi \sigma_{m} \sigma_{n} \sqrt{1-\rho^{2}}} \times e^{-\frac{1}{2\left(1-\rho^{2}\right)}\left[\frac{\left(m-\mu_{m}\right)^{2}}{\sigma_{m}^{2}}-2 \rho\left(\frac{m-\mu_{m}}{\sigma_{m}}\right)\left(\frac{n-\mu_{n}}{\sigma_{n}}\right)+\frac{\left(n-\mu_{n}\right)^{2}}{\sigma_{n}^{2}}\right]},
$$

where the variables $\mu$ denotes mean, variable $\rho$ denotes correlation coefficient, and variable $\sigma$ denotes standard deviation. In this work, the $\sigma=1.3$ and $\rho=1$. 


\section{Proposed Inverse Halftoning Technique Based on Bayesian}

\section{Theorem}

In this section, the proposed inverse halftoning method is explained in detail as below, and the algorithm is shown in Fig. 1.

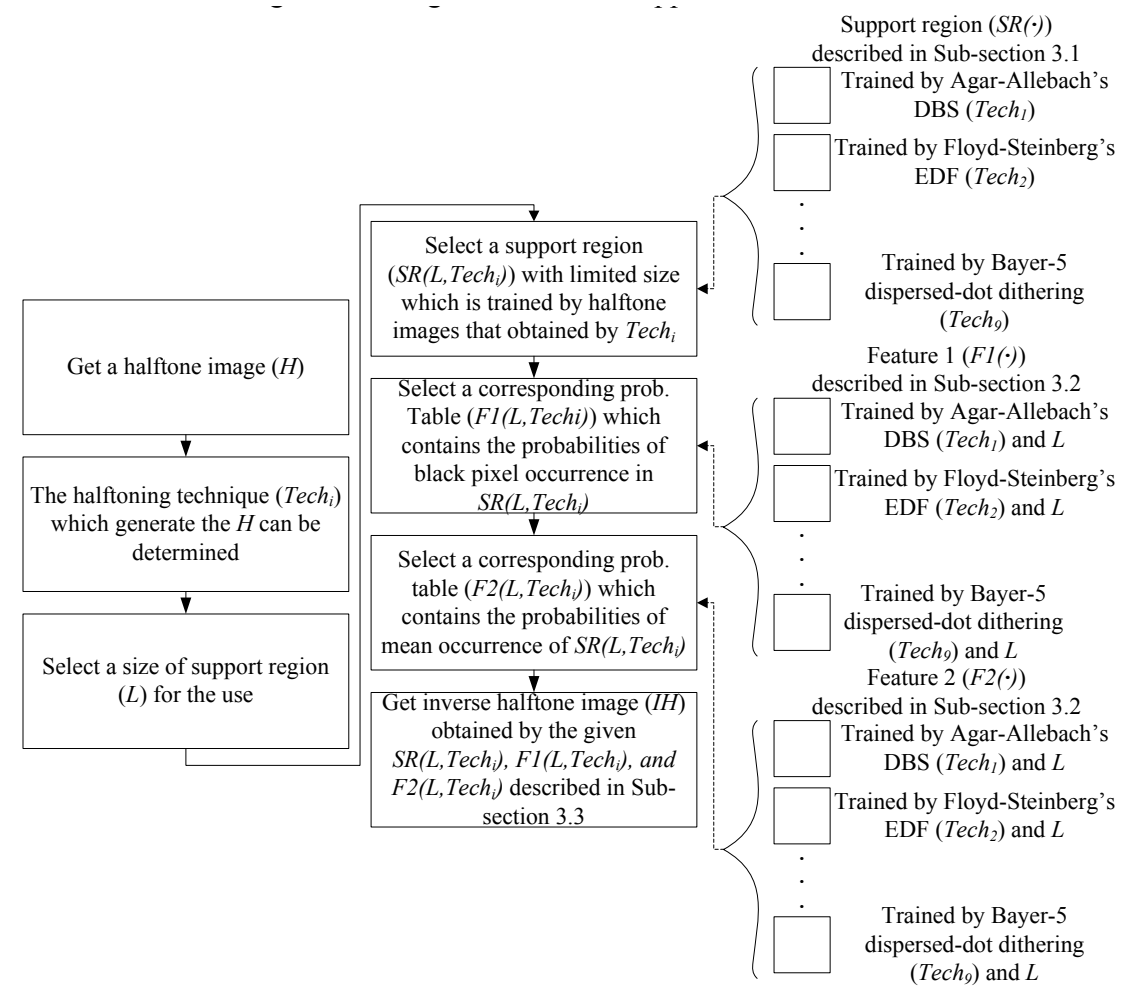

Fig. 1. Flow chart of the proposed inverse halftoning algorithm

\subsection{Support Region}

The support region refers to the referenced region on $H$ to obtain a grayscale value of current processing pixel. The center of this region is current processing position. In practice, a way to derive the support region is to use a training set of pairs of grayscale images and its corresponding halftone results. In this work, an iteration-based halftoning DBS, three kinds of EDF and DD, and two kinds of OD are adopted to produce the set. The LMS is used to derive the importance at each position in $S R$ as described as follows, 


$$
\begin{gathered}
\hat{h}_{i, j}=\sum_{m, n \in S R(15 \times 15)} \sum_{e_{i, j}^{2}=\left(\text { ori }_{i, j}-\hat{h}_{i, j}\right)^{2},} h_{i+m, j+n}, \\
\frac{\partial e_{i, j}^{2}}{\partial w_{m, n}}=-2 e_{i, j} h_{i+m, j+n}, \\
\left\{\begin{array}{c}
w_{m, n} \text { should be decreased, if } w_{m, n}>w_{m, n, o p t}, \frac{\partial e_{i, j}^{2}}{\partial w_{m, n}}>0 \\
w_{m, n} \text { should be increased, if } w_{m, n}<w_{m, n, o p t}, \frac{\partial e_{i, j}^{2}}{\partial w_{m, n}}<0
\end{array},\right. \\
w_{m, n}^{k+1}=w_{m, n}^{k}+\mu e_{i+m, j+n} h_{i+m, j+n},
\end{gathered}
$$

where variable $h_{i, j}$ denotes halftone value at current processing position $(i, j)$ on $H$. The variables $w_{m, n}$ and $w_{m, n, o p t}$ denote the weight at position $(m, n)$ in LMS-trained filter $(W)$ and optimum LMS-trained filter $\left(W_{o p t}\right)$, where the region size are the same as support region $S R$. The variable $e_{i, j}^{2}$ denotes the mean-square-error (MSE) between ori $i_{i, j}$ and $\hat{h}_{i, j}$, and variable $\mu$ denotes the adjusting parameter used to control the convergent speed of LMS optimization procedure. In this work, the variable $\mu$ is set to be $10^{-5}$ and $S R$ is fixed at size $15 \times 15$. Notably, these obtained $W_{\text {opt }}$ have some basic HVS characteristics: 1) the diagonal has less sensitivity than that of vertical and horizontal directions, and 2) the center portion has the highest sensitivity and it decreases while moving away from the center. Finally, the support region $S R\left(\right.$ Tech $\left._{i}\right)$ can be obtained by relabeling the weight by order of importance in $W_{\text {opt }}$. The $W_{\text {opt }}$ is obtained by different trained halftone images which obtained by $T e c h_{i}$.

In digital images, the obtained grayscale value $i h_{i, j}$ of current processing position $(i, j)$ is affected by halftone values from neighboring pixels, and the neighbor position with higher importance degree will increase the influence degree for $i h_{i, j}$. On the other hand, the $i h_{i, j}$ is the joint result from all pixels in the support region, and the accurate value is affected by the proportion between the number of black pixels and white pixels in support region. For example, if the number of black pixels is higher than white pixels, then $i h_{i, j}$ tends to 0 . On opposite side, the $i h_{i, j}$ tends to 255. According this phenomenon, the relationship between the represented gray levels $\left(g_{R}\right)$ and the corresponding black and white pixel numbers can be expressed by following equation:

$$
g_{R}=\operatorname{round}\left(\frac{N_{w} \times 255+N_{b} \times 0}{N_{w}+N_{b}}\right),
$$

where variable $N_{w}$ and $N_{b}$ denote the number of required white and black pixels for representing the gray level $g_{R}$. The function $\operatorname{round}(\cdot)$ represents rounding off to the nearest whole number. The required total number of pixels which is obtained from $N_{w}+N_{b}$ represents the required size of support region. Since the required gray level can be obtained by the same proportion of number of black to white pixels, some gray 


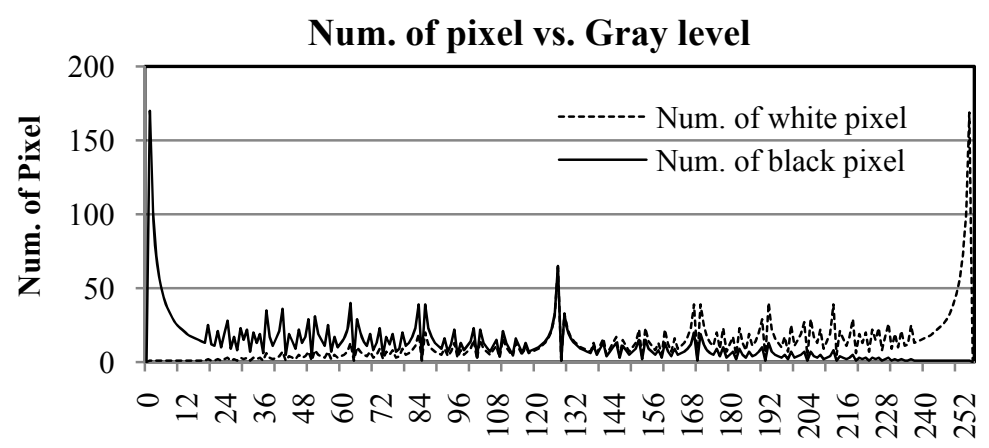

Gray level

Fig. 2. The minimum required numbers of used pixels for express each gray level

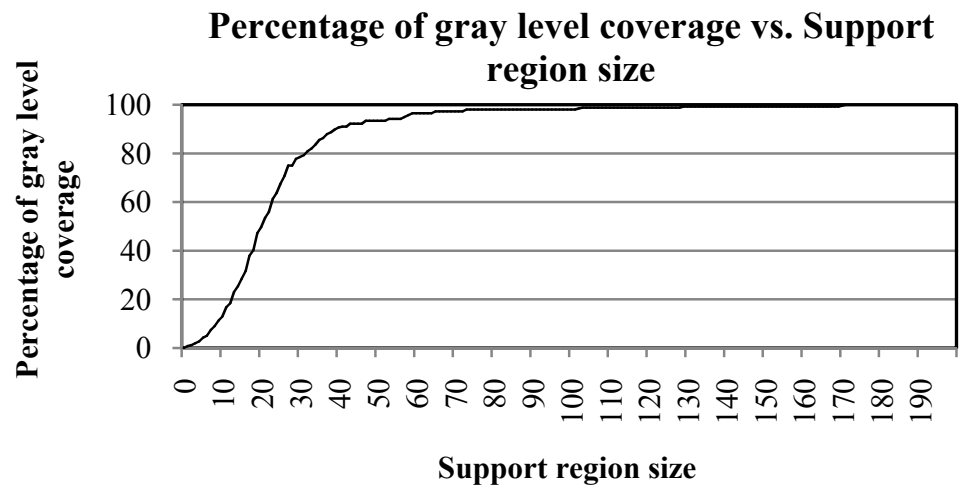

Fig. 3. Number of representable gray level subjected to a limited support region size

levels may have many different combinations of black and white pixels. This number of combinations together with the size of the support region affects the level of memory consumption. Hence, simulation for the minimum number of pixels used to express each gray level is required and is detailed in Fig. 2. According to this figure, bigger support region sizes can express more gray levels. The relationship between percentage of $g_{R}$ and the support region size is shown in Fig. 3, where the sizes from 13 to 27 have the huge variations. The flat regions mean that there are no additional representable gray levels when the size of support region is increased in that region. When the size of support region is increased to 171, all gray levels can be represented.

\subsection{Features}

Based on the concept of smoothing characteristic of HVS and processing manner of halftoning, the neighboring pixels around the current processing position $(i, j)$ will 
affect $i h_{i, j}$. According to this concept, two features are used: 1) the probability of black pixel occurrence at each position in the support region, and 2) the probability of mean occurrence which is obtained from all pixels in the support region.

In the training procedure to obtain these features, 153 natural training images of size $512 \times 512$ were used. Firstly, select a halftoning technique Tech $_{i}$ and corresponding $S R\left(L\right.$, Tech $\left._{i}\right)$ with limited support region size $L$. For feature 1 ( F1(·)), Mese-Vaidyanathan's inverse halftoning [12] checks the combinations of black and white pixels in the support region with limited $L$ to find the grayscale value of the current processing pixel. The memory consumption of this method increases by $2^{L}$. Which means this method requires much memory. To solve this problem, it is replaced by the probability of black pixel occurrence at each position in the support region. The memory consumption is thus reduced to $N_{g_{R}} \times L$, where variable $N_{g_{R}}$ denotes the number of $g_{R}$ in limited $L$. The probability is described as below,

$$
\begin{aligned}
& p\left(h_{i+m, j+n}=0 \mid \operatorname{ori}_{i, j}\right) \\
& =\frac{\operatorname{Num}\left(h_{i+m, j+n}=0 \mid \operatorname{ori}_{i, j}\right)}{\operatorname{Num}\left(h_{i+m, j+n}=0 \mid \operatorname{ori}_{i, j}\right)+\operatorname{Num}\left(h_{i+m, j+n}=255 \mid \text { ori }_{i, j}\right)},
\end{aligned}
$$

where position $(m, n) \in S R\left(L\right.$, Tech $\left._{i}\right)$, variable $\operatorname{ori}_{i, j} \in g_{R}$, and function $\operatorname{Num}(\cdot)$ denotes that calculate an occurred counts for a given event. In the explanation of Sub-section 3.1, $N_{g_{R}}$ cannot be 256 except the case when $L$ exceeds 171 . When ori $_{i, j} \in g_{R}$ then the statistical summary can be obtained by Eq. (9). Reversely, the sample will be excluded from this training procedure since that may affect the correctness of the original information.

For feature $2(F 2(\cdot))$, the amount of required memory is $N_{g_{R}}{ }^{2}$, which is also affected by $L$. The probability is described as follows,

$$
p\left(m_{p} \in g_{R} \mid \operatorname{ori}_{i, j} \in g_{R}\right)=\frac{\operatorname{Num}\left(m_{p} \in g_{R} \mid \operatorname{ori}_{i, j} \in g_{R}\right)}{\operatorname{Num}\left(\operatorname{ori}_{i, j} \in g_{R}\right)},
$$

where the variable $m_{p}$ denotes the mean is $p$. In the trained probabilistic tables of means, where empty occurs when the trained halftone images only have one type (e.g., only ordered dithering). This is because the combinations of halftone values of each pixel in the support region concentrate on some combinations when the halftone image is observed from a reduced region. As this may occur when the sample is not enough, the $p\left(m_{p} \in g_{R} \mid\right.$ ori $\left._{i, j} \in g_{R}\right)$ is remodeled based on original data once again by Gaussian distribution in the following manner.

$$
\begin{gathered}
G(x \mid \mu, \sigma)=\frac{1}{\sigma \sqrt{2 \pi}} e^{-\frac{(x-\mu)^{2}}{2 \sigma^{2}}}, \\
p\left(m_{p} \in g_{R} \mid \operatorname{ori}_{i, j} \in g_{R}\right)=\frac{\sum_{k=-3}^{3} p\left(m_{p+k} \in g_{R} \mid o r i_{i, j} \in g_{R}\right) G(k \mid \mu, \sigma)}{\sum_{k=-3}^{3} G(k \mid \mu, \sigma)},
\end{gathered}
$$

where function $G(\cdot)$ is a 1-D Gaussian distribution. The variable $\sigma=1$ and size of Gaussian distribution is $7 \times 7$ in this work. 


\subsection{Inverse Halftoning Technique Based on Bayesian Theorem}

The performance of inverse halftoning techniques are determined by the degree of similarity between obtained $I H$ and Ori under limited information. Bayesian theorem is a fundamental probabilistic approach to the problem of classification, which can get the optimum decision based on all the probabilistic features known. Based on these features, the probability of the various grayscale values at the position currently being processed can be determined. The conditional probability is described as following,

$$
p\left(g_{q} \mid h_{0}, h_{1}, \ldots, h_{L-1}, m\right)=\frac{p\left(g_{q} \cap h_{0}, h_{1}, \ldots, h_{L-1}, m\right)}{p\left(h_{0}, h_{1}, \ldots, h_{L-1}, m\right)},
$$

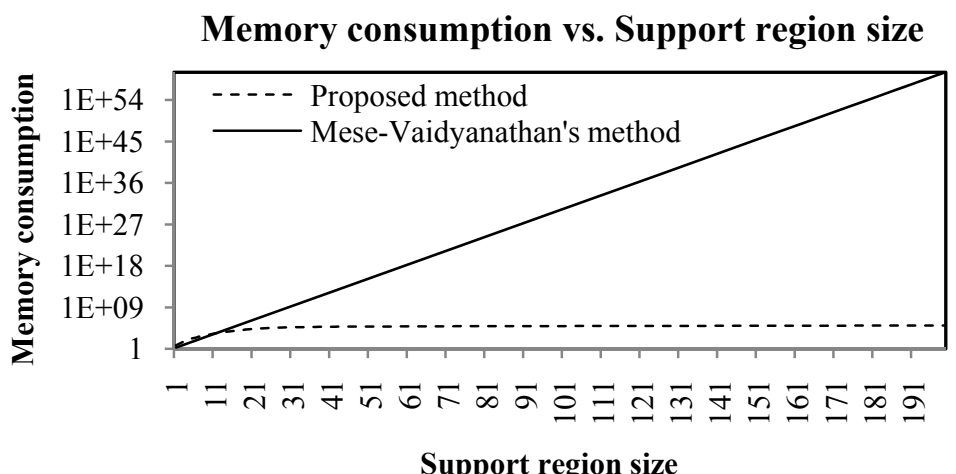

(a)

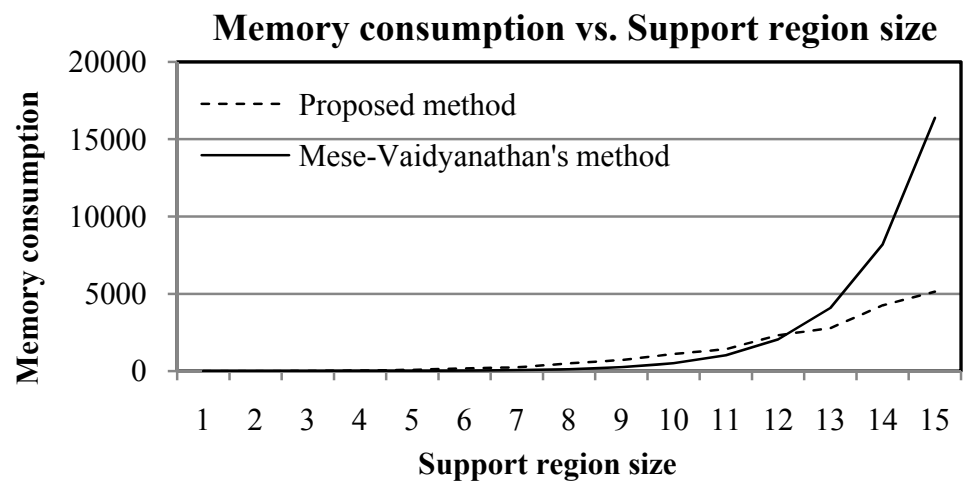

(b)

Fig. 4. The comparison of memory consumption between proposed method and MeseVaidyanathan's method [12]. (a) The vertical axis is compressed by $\log (\cdot)$. (b) Reduced the range of horizontal axis from 1 to 15 . 


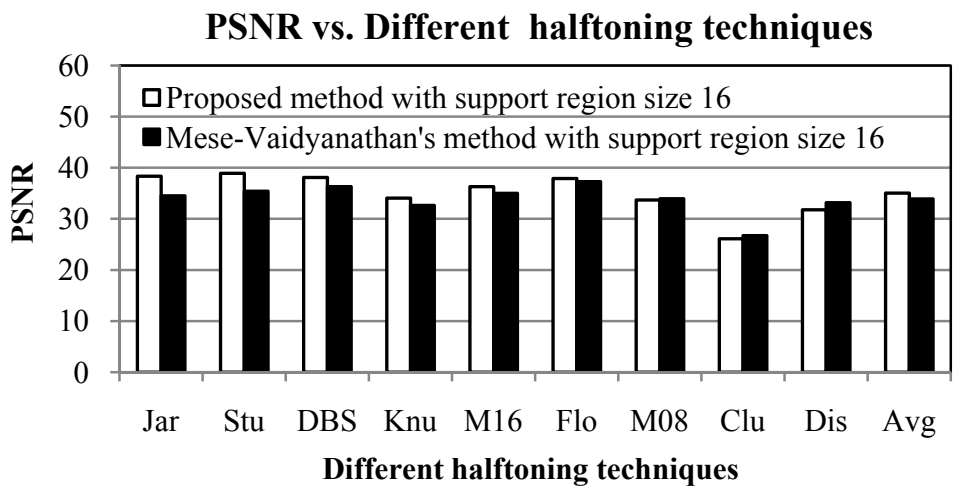

Fig. 5. Average PSNR of different 202 natural images obtained from different halftone images

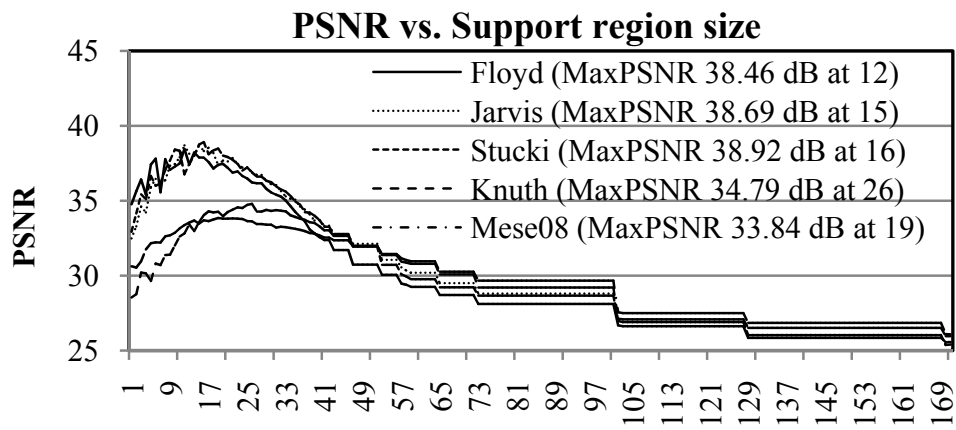

Support region size

(a)

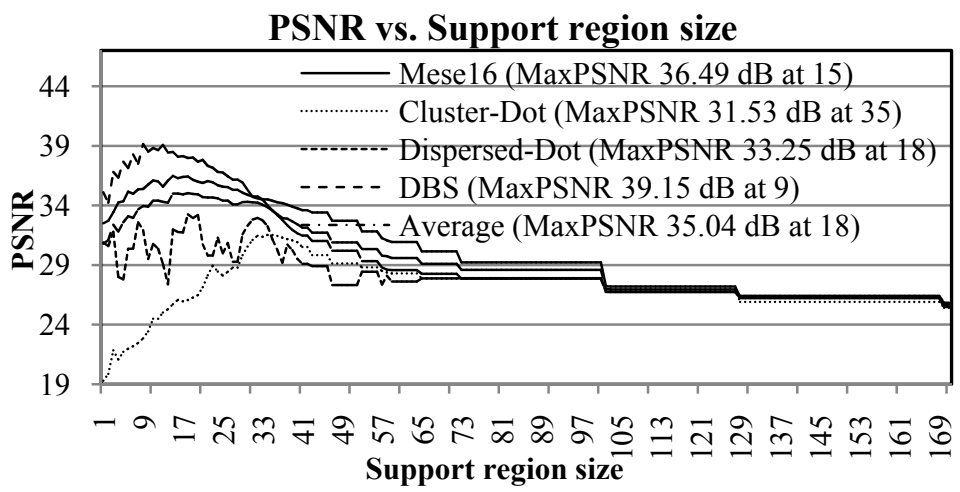

(b)

Fig. 6. Average PSNR of different kinds of halftoning techniques for different support region size from 1 to 171 
where variable $g_{q}$ denotes grayscale value $q$, where $q \in g_{R}$, variable $h_{k}$ denotes the halftone value of neighbor position with $k$ th importance order in $S R\left(L, T e c h_{i}\right)$, and variable $m$ denotes the mean of these pixels in $S R\left(L, T e c h_{i}\right)$. Focus on numerator, the above equation can be rewrite as following,

$$
p\left(g_{q} \mid h_{0}, h_{1}, \ldots, h_{L-1}, m\right)=\frac{p\left(g_{q}\right) p\left(h_{0}, h_{1}, \ldots, h_{L-1}, m \mid g_{q}\right)}{p\left(h_{0}, h_{1}, \ldots, h_{L-1}, m\right)},
$$

where $p\left(g_{q}\right)$ is called priori probability, $p\left(h_{0}, h_{1}, \ldots, h_{L-1}, m \mid g_{q}\right)$ called likelihood of $g_{q}$ with respect to these features $h_{0}, h_{1}, \ldots, h_{L-1}, m$, and $p\left(h_{0}, h_{1}, \ldots, h_{L-1}, m\right)$ called the evidence. The memory consumption of numerator is ${N_{g_{R}}}^{2} \times 2^{L}$, where the $p\left(g_{q}\right)$ is supposed $\frac{1}{N_{g_{R}}}$ in this work, and the other part are used for searching the mapping probability of $p\left(h_{0}, h_{1}, \ldots, h_{L-1}, m \mid g_{q}\right)$. The above equation is rewritten for letting all features are independently as below,

$$
p\left(g_{q} \mid h_{0}, h_{1}, \ldots, h_{L-1}, m\right)=\frac{p\left(g_{q}\right) p\left(m \mid g_{q}\right) \prod_{k=0}^{L-1} p\left(h_{k} \mid g_{q}\right)}{p\left(h_{0}, h_{1}, \ldots, h_{L-1}, m\right)} .
$$

For this, the memory consumption is reduced to ${N_{g_{R}}}^{2}+N_{g_{R}} \times L$. For the evidence, it is probably changed when $H$ is different from the first trained halftone image. For this reason, the evidence re-described by Bayesian theorem as follows,

$$
p\left(g_{q} \mid h_{0}, h_{1}, \ldots, h_{L-1}, m\right)=\frac{p\left(g_{q}\right) p\left(m \mid g_{q}\right) \prod_{k=0}^{L-1} p\left(h_{k} \mid g_{q}\right)}{\sum_{r \in g_{R}}\left[p\left(g_{r}\right) p\left(m \mid g_{r}\right) \prod_{k=0}^{L-1} p\left(h_{k} \mid g_{r}\right)\right]} .
$$

Besides, the probabilities of all $g_{R}$ are obtained. Finally, the $i h_{i, j}$ can be obtained by following equation,

$$
i h_{i, j}=\underset{q \in g_{R}}{\operatorname{argmax}}\left[p\left(g_{q} \mid h_{0}, h_{1}, \ldots, h_{L-1}, m\right)\right]
$$

According this method, the $I H$ can be obtained.

\section{Experimental Results}

In this section we apply the proposed Bayesian-based inverse halftoning for quantitative evaluation. Focus on the comparison of memory consumption with Mese-Vaidyanathan's inverse halftoning [12] is shown in Fig. 4. This figure represents the amount of required memory at different support region size $L$. The vertical axis of Fig. 4(a) is compressed by log function since we can perceive the memory consumption of Mese-Vaidyanathan's method to be increased by power law. The memory consumption of the proposed method with 171 support region size that can express all the 


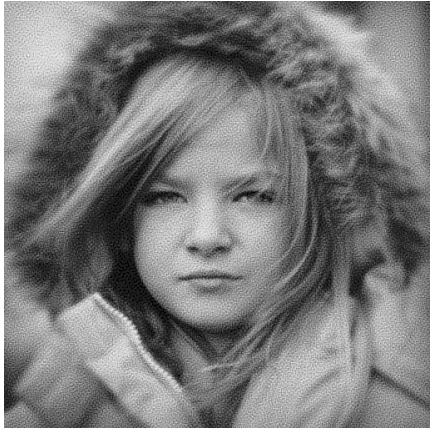

(a) PSNR $=40.01 \mathrm{~dB}$

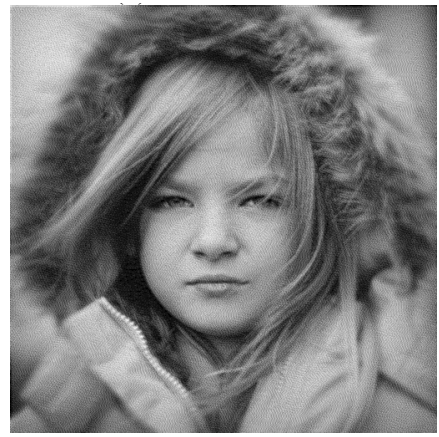

(c) PSNR $=40.22 \mathrm{~dB}$

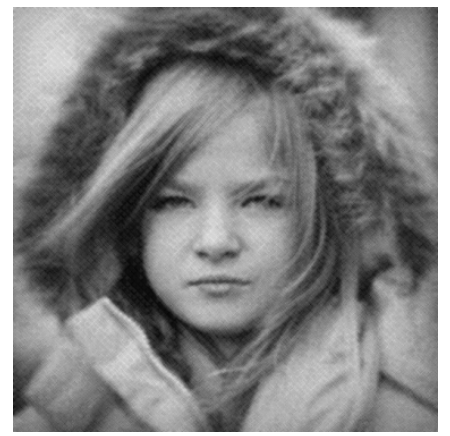

(e) PSNR $=36.62 \mathrm{~dB}$

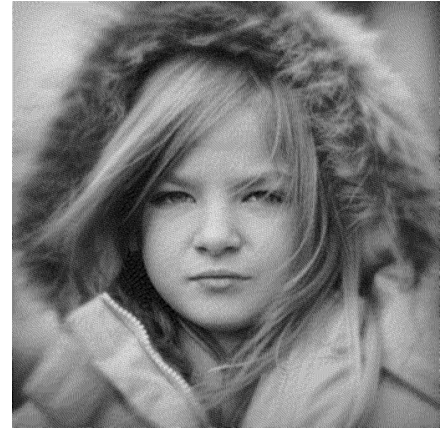

(b) PSNR $=40.61 \mathrm{~dB}$

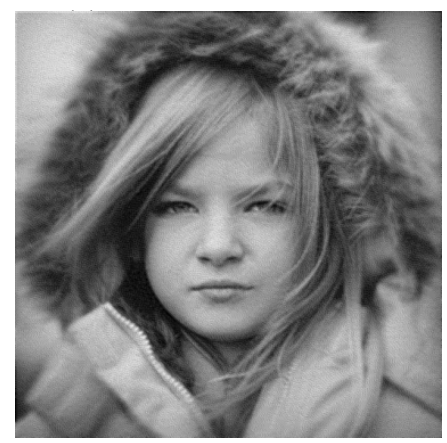

(d) PSNR $=40.77 \mathrm{~dB}$

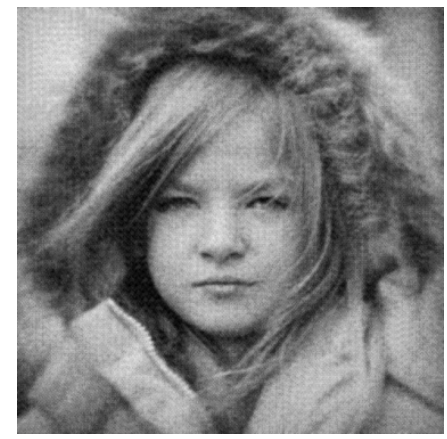

(f) $\mathrm{PSNR}=34.13 \mathrm{~dB}$

Fig. 7. Inverse halftone images using the proposed method with the corresponding optimum support region size. (a) DBS [12] with $L=9$. (b) EDF [4] with $L=12$. (c) EDF [5] with $L=15$. (d) EDF [6] with $L=16$. (e) DD [2] with $L=26$. (f) DD [3] with class matrix of size $8 \times 8$ and $L=19$, and (g) with class matrix of size $16 \times 16$ and $L=15$. (h) OD [1] Classical-4 cluster-dot dithering with $L=35$, and (i) Bayer-5 dispersed-dot dithering with $L=18$. (all printed at 300dpi). 


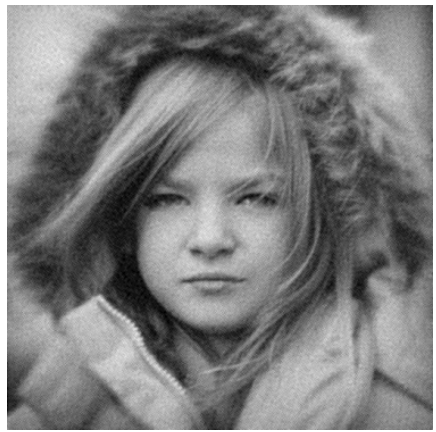

(g) PSNR $=38.07 \mathrm{~dB}$

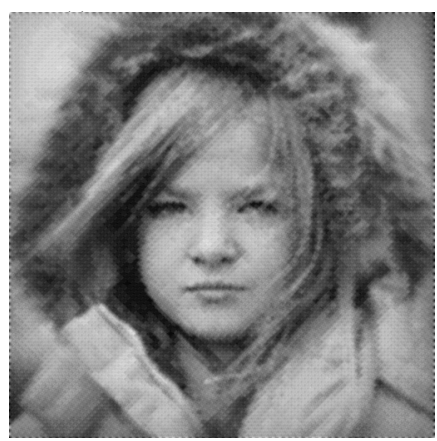

(h) PSNR $=32.84 \mathrm{~dB}$

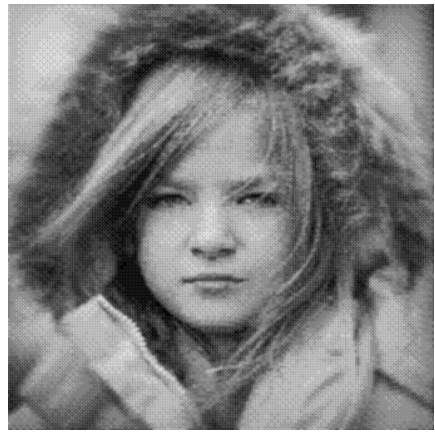

(i) $\mathrm{PSNR}=33.57 \mathrm{~dB}$

Fig. 7. (continued)

gray level is 109312. Focus on the range of different support region sizes from 1 to 15 is shown in Fig. 4(b), the memory consumption of Mese-Vaidyanathan's method is superior to the proposed method in range from 1 to 12 . However, it cannot provide better inverse halftone image quality for different halftoning techniques with support region size in this small.

Figure 5 shows the comparison of image quality between Mese-Vaidyanathan's method and the proposed method. Among these results, 202 testing images of size $512 \times 512$ were used to derive the average PSNR. After which, 9 different halftoning techniques were taken into account, which include Agar-Allebach's DBS [10], error diffusion as Floyd-Steinberg's method [4], Jarvis et al.'s method [5], and Stucki's method [6], dot diffusion as Knuth's method [2] and Mese-Vaidyanathan's method [3], and ordered dithering as Ulichney's Classical-4 clustered-dot and Bayer-5 dispersed-dot [1]. The average PSNRs of Mese-Vaidyanathan's method and proposed method are $33.87 \mathrm{~dB}$ and $35.01 \mathrm{~dB}$, respectively.

Figure 6 shows the average PSNR of different kinds of halftoning techniques with different support region sizes using the proposed method. According to these results, the halftoning techniques which can obtain better quality of halftone image have smaller optimal support region size. The region on the tail of curve is flat since the 
number of representable gray levels is fixed when the support region is increased as shown in Fig. 3. The optimum support region sizes for different halftoning techniques are concentrated in the range from 9 to 35 , because the smoothing degree is directly proportional with support region size. Finally, the resulting inverse halftone images obtained by proposed method of different halftoning techniques with corresponding optimum support region size are shown in Fig. 7.

\section{Conclusions}

An inverse halftoning which based on Bayesian theorem is proposed. Two features are taken into account: 1) the probability of black pixel occurrence for each position in the support region, and 2) the probability of mean occurrence which is obtained from all pixels in the support region. Throughout this work, nine different kinds of halftoning were used for evaluating the performance of the proposed method. According to the experimental results, the image quality of proposed method is superior to Mese-Vaidyanathan's inverse halftoning. For Mese-Vaidyanathan's inverse halftoning, when the original halftone images have better image quality, such as error diffusion, DBS, and dot diffusion except Mese-Vaidyanathan's dot diffusion with $8 \times 8$ class matrix, the expression on the ramp low frequency part of inverse halftone results is inferior to proposed method. Furthermore, in terms of memory consumption, the proposed method is inferior to Mese-Vaidyanathan's inverse halftoning when the size of support region is from 1 to 12 . However, the optimum support region size of all halftoning techniques in experimental results is always bigger than 12 which are except the halftoning technique DBS. In future work, a technique for classifying different kinds of halftoning will be developed.

\section{References}

1. Ulichney, R.: Digital Halftoning. MIT Press, Cambridge (1987)

2. Knuth, D.E.: Digital halftones by dot diffusion. ACM Trans. Graph. 6(4) (October 1987)

3. Mese, M., Vaidyanathan, P.P.: Optimized halftoning using dot diffusion and methods for inverse halftoning. IEEE Trans. on Image Processing 9, 691-709 (2000)

4. Floyd, R.W., Steinberg, L.: An adaptive algorithm for spatial gray scale. In: Proc. SID 75 Digest. Society for information Display, pp. 36-37 (1975)

5. Jarvis, J.F., Judice, C.N., Ninke, W.H.: A survey of techniques for the display of continuous-tone pictures on bilevel displays. Comp. Graph. Image Proc. 5, 13-40 (1976)

6. Stucki, P.: MECCA-A multiple-error correcting computation algorithm for bilevel image hardcopy reproduction. Res. Rep. RZ1060, IBM Res. Lab., Zurich, Switzerland (1981)

7. Ostromoukhov, V.: A simple and efficient error-diffusion algorithm. In: Computer Graphics (Proceedings of SIGGRAPH 2001), pp. 567-572 (2001)

8. Shiau, J.N., Fan, Z.: A set of easily implementable coefficients in error diffusion with reduced worm artifacts. In: SPIE, vol. 2658, pp. 222-225 (1996) 
9. Lin, Q., Allebach, J.P.: Color FM screen design using DBS algorithm. In: Proc. SPIE, vol. 3300, pp. 353-361 (1998)

10. Agar, A.U., Allebach, J.P.: Model-based color halftoning using direct binary search. IEEE Trans. on Image Processing 14, 1945-1959 (2005)

11. Chang, P.-C., Yu, C.-S.: Neural net classification and LMS reconstruction to halftone images. In: Proc. SPIE, vol. 3309, pp. 592-602 (1998)

12. Mese, M., Vaidyanathan, P.P.: Look-Up Table (LUT) Method for Inverse Halftoning. IEEE Trans. on Image Processing 10(10), 1566-1578 (2001) 